\title{
Una reforma global de la formación de los profesionales sanitarios
}

\author{
Andreu Segura-Benedicto
}

Cada año en el mundo se forma un millón de nuevos profesionales sanitarios en las 2.420 facultades de medicina y las 467 escuelas de salud pública, junto a un número indeterminado de escuelas de enfermería, con un coste total de unos cien mil millones de dólares anuales, lo que viene a representar algo menos del $2 \%$ del gasto sanitario global. Una proporción baja para una actividad tan dependiente de los recursos humanos empleados, y con una gran disparidad entre países. Estos datos se incluyen en el informe de una comisión independiente de la revista The Lancet, en el que se denuncia la poca información disponible sobre la formación de los profesionales, aunque nadie ponga en duda que es uno de los elementos primordiales, si no el que más, a la hora de conseguir de los sistemas sanitarios la eficiencia y equidad que la atención sanitaria de la población mundial necesita.

A principios de abril del pasado año, dicha revista solicitaba de los lectores su colaboración en un estudio emprendido por una nueva comisión sobre la formación de los profesionales sanitarios en el siglo Xxi. Creada bajo los auspicios de la revista y la ayuda inicial de las fundaciones de Bill \& Melinda Gates y Rockefeller, además del China Medical Board, su propósito principal es fomentar una reforma global de la formación de los profesionales, necesaria debido a una serie de poderosas razones entre las que destacan, según los propios comisionados [1], los profundos cambios demográficos, sociológicos, económicos y tecnológicos -incluyendo tanto los avances en sistemas de información y comunicación como las innovaciones más específicamente sanitarias-, todo lo cual requiere nuevas aproximaciones educativas.

También señalan las limitaciones de las recientes iniciativas para mejorar la salud mundial, que no han conseguido sus objetivos, probablemente porque no se ha prestado suficiente atención a la importancia de los recursos humanos como elemento fundamental para que los sistemas sanitarios funcionen adecuadamente. Si bien se han dedicado notables esfuerzos para corregir las carencias cuantitativas, hay que considerar también los aspectos cualitativos, particularmente aquellos que se refieren a la capacidad de liderazgo de los profesionales en todos los ámbitos de la sanidad. En este sentido, la diferencia entre las actuales competencias profesionales y las que demanda un mundo cada vez más interdependiente exigen una adaptabilidad en la formación que permita funcionar local y globalmente. Ello pone en evidencia el anquilosamiento de numerosos planes y programas formativos, que nacieron ya anticuados al concebirse sin la ambición necesaria, todo lo cual comporta graduados mal preparados por instituciones pobremente financiadas.

Echan en falta una deliberación pública sobre las dimensiones globales de las necesidades y demandas sanitarias y la inexistencia de nuevos argumentos y de estimulantes reflexiones individuales e institucionales sobre la salud. Ello constituye una rémora para aprovechar las oportunidades emergentes en el ámbito de la formación de los profesionales sanitarios -desarrollo de competencias basadas en las destrezas, aprendizaje a distancia mediante las tecnologías de la información y la comunicación, aprendizaje interprofesional y en equipo, nuevas modalidades que relacionan la investigación y la docencia desde el aula a la práctica real, y nuevas posibilidades de colaboración en todo el mundoque sirvan para equiparlos con las herramientas, los conocimientos y la sensibilidad que permitan mejorar su efectividad.

La verdad es que la comisión se ha tomado con diligencia el encargo porque a principios de diciembre [2] ya se publicaban los resultados cuyas conclusiones principales remiten a diez recomendaciones, seis referidas a las modificaciones en la naturaleza de la formación, que los autores califican como de instrucción, y otras cuatro denominadas institu-
Área de Salud Pública. Institut d'Estudis de la Salut. Departamento de Ciencias Experimentales y de la Salud. Universitat Pompeu Fabra. Barcelona, España.

Correspondencia: Prof. Andreu Segura Benedicto. Área de Salud Pública. Institut d'Estudis de la Salut. Roc Boronat, 81-95. E-08005 Barcelona.

E-mail: andreu.segurab@gencat.cat (c) 2011 Educación Médica 
cionales, acerca del papel que deben desempeñar las facultades y escuelas de profesionales sanitarios en el contexto internacional. Estas reformas, mediante un proceso de intervención que incluye cuatro actividades básicas -estimular el liderazgo, mejorar las inversiones, acreditar la formación y fortalecer el aprendizaje global-, deberán conducir a la meta, que no es otra que convertir la formación en un elemento transformador e interdependiente para conseguir la equidad en la salud, es decir, la eliminación de las desigualdades injustas y evitables.

Las reformas relativas a la instrucción abarcan todo el recorrido entre la admisión y la graduación, con el propósito de generar programas basados en las competencias profesionales que, mediante el uso creativo de las nuevas tecnologías de la información y la comunicación, preparen efectivamente a los estudiantes para el trabajo en equipo, capaces de evolucionar flexiblemente y en base al espíritu de un nuevo profesionalismo. De forma breve, incluyen la adopción de un currículo basado en las competencias profesionales necesarias para responder flexiblemente a las necesidades de intervención -que a su vez también cambian rápidamente-; la promoción de una formación inter y transprofesional que acabe con la configuración predominante de las profesiones en compartimentos estancos, lo que obstaculiza el trabajo en equipo; el uso intensivo de las posibilidades de las tecnologías de la información y la comunicación; la adaptación a las necesidades y problemas locales, pero sin dejar de aprovechar los recursos globales en forma de conocimientos, experiencias, materiales didácticos e intercambio de estudiantes; el fortalecimiento de los recursos educativos mediante inversiones, evaluación y desarrollo del profesorado y de los equipamientos y procedimientos; y, finalmente, la promoción de un nuevo profesionalismo que utilice las competencias como el criterio objetivo de clasificación de los profesionales sanitarios, dejando a un lado las categorías actuales, impermeables entre sí. El profesionalismo que se necesita se basa en las actitudes, valores y comportamientos que, como un complemento al aprendizaje de las destrezas correspondientes, permitan actuar como agentes de cambio que dan cuenta de lo que consiguen, como competentes gestores de recursos y promotores de políticas basadas en pruebas científicas.

Las reformas institucionales pretenden mejorar el rendimiento de los esfuerzos nacionales mediante sinergias como la planificación conjunta, sobre todo en los sectores de la educación y la sanidad, una responsabilidad que no se comparte con la frecuencia que sería aconsejable, para involucrar a todos los agentes implicados en el proceso de reforma, desarrollar redes de colaboración global para el apoyo mutuo y fomentar una cultura de indagación crítica y de transparencia; conseguir establecer sinergias que multipliquen los resultados de los esfuerzos de todos los agentes implicados; expandir los centros académicos en sistemas que penetren tanto en los equipamientos sanitarios como en las comunidades; relacionar las instituciones educativas con los agentes sociales y económicos mediante redes, alianzas y consorcios; y, finalmente, alimentar una cultura de abierto pensamiento crítico, en la que resulta crucial el desarrollo del conocimiento científico, la deliberación ética y el debate público y razonado que permita una transformación social ilustrada.

El trabajo, denso y extenso, se estructura en tres secciones y viene precedido de un resumen ejecutivo. La primera sección describe el problema a partir de un resumen panorámico de la evolución de la formación de los profesionales sanitarios en el último siglo, destacando el centenario de la publicación del informe Flexner. Esta efemérides también la conmemoraba la fundación Carnegie, institución que patrocinó el transcendental trabajo de Abraham Flexner mediante el cual se consolidó la orientación científica adoptada por la mayoría de los planes de estudio de las facultades de medicina [3]. Más que un recuerdo, constituye la referencia desde la que proponer, a su vez, un cambio radical de la formación de los profesionales sanitarios, particularmente de los médicos [4], una transformación que coincide con la propuesta de Frenk et al en muchos aspectos, algunos más tradicionales -como la integración de teoría y práctica- y otros más modernos -como el desarrollo del espíritu crítico-. También comparten el fomento del profesionalismo, que conlleva unas actitudes consecuentes con los valores de la cultura de la responsabilidad y la libertad, aunque en el caso de la comisión de The Lancet se insista en un nuevo planteamiento que supere la estanqueidad de las profesiones sanitarias actuales.

En la segunda sección se muestran los principales resultados de los trabajos de la comisión, entre los que destaca la primera valoración cuantitativa de las instituciones educativas en medicina, enfermería y salud pública en el mundo, complementada con un primer análisis de la heterogeneidad de su distribución geográfica -medida según la densidad de centros educativos por cada diez millones de habitantes- y de la inversa relación entre el número de centros y el de profesionales respecto de la carga de enfermedad -medida en años de vida ajustados por discapacidad por cien mil-, o las enormes disparidades en términos económicos que suponen las 
inversiones y los costes de producción de nuevos titulados. En esta sección se consideran los sistemas y procedimientos de acreditación que, en el ámbito de las profesiones sanitarias, resulta crucial, dado que es imprescindible para legitimar formalmente a las instituciones educativas, pero que no existe en una cuarta parte de los países del mediterráneo oriental, en la mitad de los del sudeste asiático y en dos terceras partes de los países africanos. La valoración de estos procedimientos es de gran interés porque la calidad de la acreditación refleja, al menos en parte, la incorporación social del profesionalismo, lo que nos lleva a considerar la responsabilidad social de las profesiones y la necesidad de transparencia y de rendir cuentas a las poblaciones. Ello constituye uno de los aspectos de mayor enjundia política porque el principal reto que afronta la formación de los profesionales sanitarios en este nuevo siglo es precisamente el de contribuir de forma efectiva a la mejora del funcionamiento de los sistemas sanitarios y a la de la salud de la población [5].

Como se señala en el último apartado del informe, el 'camino a seguir', el primer paso, debe ser un cambio en la mentalidad que nos permita reconocer los problemas y buscar las soluciones. La reforma será un proceso largo y difícil que demanda un potente liderazgo y requiere cambiar las actitudes, los estilos de trabajo y una buena relación entre los agentes implicados. De ahí la importancia de preguntarse acerca de la vigencia de algunos paradigmas que la medicina y la sanidad han incorporado de forma poco crítica, como por ejemplo, los que han dado lugar a la creciente medicalización de la vida cotidiana, con algunas ventajas pero con notables inconvenientes; entre éstos, el elevado coste de las intervenciones no es el más importante, ya que el consumo sanitario inapropiado induce un incremento considerable de la yatrogenia, uno de los principales problemas de salud pública, sobre todo en los países más ricos.

Así pues, conviene considerar cuidadosamente las indicaciones y las limitaciones de las intervenciones clínicas y sanitarias, de modo que las extraordinarias posibilidades que abre el progreso del co- nocimiento y el desarrollo de la tecnología actúen efectivamente en provecho de las personas y de las comunidades.

En el ámbito de las instituciones educativas, es de interés mencionar la experiencia de la asociación de profesionales y centros denominada 'The Network: Towards Unity for Health', citada también en el informe de la comisión. Se trata de una plataforma global para el fomento de la equidad sanitaria mediante una orientación formativa basada en la comunidad, modelos de investigación participativa y trabajo en red, de la que forman parte iniciativas locales como el proyecto AUPA (Actuando Unidos para la Salud), que se viene desarrollando desde hace unos años en Cataluña y en el que colaboran los servicios sanitarios colectivos de la salud pública, 60 equipos de atención primaria -es decir, un $15 \%$ de los existentes- y un conjunto de entidades cívicas y ciudadanas.

La actuación integrada de los dispositivos sanitarios, particularmente los que corresponden a la atención primaria de salud y los de la salud pública, en una perspectiva de salud comunitaria, puede contribuir a mejorar la efectividad, la eficiencia y la equidad de los recursos sanitarios y, lo que es todavía más importante, el 'empoderamiento' de las personas y los colectivos respecto del control de los determinantes de la salud, tal como reclamaba la carta de Ottawa hace ya 25 años.

\section{Bibliografía}

1. Bhutta ZA, Chen L, Cohen J, Crisp N, Evans T, Fineberg H, et al. Education of health professionals for the 21st century: a global independent commission. Lancet 2010; 375: 1137-8.

2. Frenk J, Chen L, Bhutta ZA, Cohen J, Crisp N, Evans T, et al. Health professionals for a new century: transforming education to strengthen health systems in an interdependent world. Lancet 2010; 376: 1923-58.

3. Cooke M, Irby DM, O'Brien BC. Educating physicians: a call of reform of medical schools and residency. The Carnegie Foundation for the Advancement of Teaching. San Francisco: Jossey-Bass; 2010.

4. Oriol-Bosch A. De Flexner a Bolonia. Educ Med 2010; 13: 193-5.

5. Boelen C, Woollard B. Social accountability and accreditation: a new frontier for educational institution. Med Educ 2009; 43: 887-94. 